\title{
A Single-hop Broadcasting Method Based on Busy Tone
}

\author{
Cao Wen-jing, Han Qing-tian, Xu Sheng-hong
}

Department of Control Engineering, Naval Aeronautical and Astronautical University, Yantai 264001, China

caowenjing751215@sohu.com

Keywords: Wireless ad hoc network, Single-hop broadcasting, Channel reservation, Busy tone

\begin{abstract}
Single-hop broadcasting is a basic information dissemination method. For broadcasting method, wireless channel reservation and channel state indication are two factors effecting information dissemination performance. In this paper, existing channel reservation and channel state indication mechanisms were studied, the limitations of them were analyzed, and a single-hop broadcasting method based on busy tone is proposed. With OPNET network simulation platform, the proposed broadcasting method is modeled and implemented. Experimental results show that the proposed method performs well.
\end{abstract}

\section{Introduction}

According to the message transferring hop counts, broadcasting methods could be classified into single-hop broadcasting and multi-hops broadcasting. For some type of messages, because of the small effected range, single-hop broadcasting could be applied to disseminate such messages. To ensure node to access channel reliably, wireless channel should be reserved. To ensure message transferring reliable, channel state should be indicated at the same time. With respect to single-hop broadcasting, channel reservation and channel state indication are two factors effecting information dissemination performance.

\section{Existing channel reservation and channel state indication mechanisms}

Existing channel reservation mechanism. Currently, the most widely used channel reservation mechanism is RTS/CTS handshake. With RTS/CTS handshake, source node and destination node interact with short control messages RTS and CTS to reserve channel before sending data message. The broadcasting protocols use RTS/CTS to reserve channel include BSMA protocol ${ }^{[1]}$, BMW protocol ${ }^{[2]}$, BMMM protocol ${ }^{[3]}$, etc. With such broadcasting protocols, source node usually handshake with each destination node one by one, therefore source node should maintain a neighbor nodes list.

RTS/CTS handshake not only notify the nodes in one-hop of source node with RTS, but also notify the nodes in two hops of source node with CTS the coming broadcasting. Therefore, RTS/CTS handshake reserves two hops range of channel of source node. In the network with high density, reserve two hops would lead to a large number of nodes waiting to access the common channel, which effect nodes access channel on time. In a word, reserve channel with RTS/CTS handshake is complex and the speed of access channel is low in high density network.

Existing channel state indication mechanism. In current broadcasting method, two hops range channel state of source node is indicated with busy tone. For example, in [4], to avoid the collisions caused by hidden terminals, at the same time of broadcasting data message, source node broadcast large range busy tone to block hidden terminal broadcasting. In high density network, two hops range or large range channel state indication would lead to a large number of nodes waiting access channel.

\section{A single-hop broadcasting method based on busy tone}

To overcome the disadvantages introduced by RTS/CTS handshake, referring to some unicast broadcasting methods, this research designed a single-hop broadcasting method based on busy tone. In this method, busy tone is used to reserve the one hop channel of source node, the broadcasted data message is used to indicate the one hop channel state of source node. 
Reserve one hop range channel with busy tone. For a node having data message to broadcast, busy tone is broadcasted to reserve one hop range channel firstly. Reserve channel lasts for time t:

$$
\mathrm{t}=\mathrm{d} / \mathrm{c}
$$

At formula (1), $d$ is one hop range of wireless signal, $c$ is the propagation velocity of wireless signal, and $t$ is the time span of wireless signal propagates one hop range. During $t$ time span, no data or busy tone from other nodes are detected means that, there is no other node in one hop range of the source node reserve channel, the source node reserve one hop range channel successfully, and could broadcast data further.

Back off mechanism. In high density network, it is more usually occurs that neighboring nodes reserve channel almost at the same time, which may lead to successive channel reservation failure and increase access channel delay greatly. Therefore, only reserve channel mechanism adopted is not enough, back off mechanism is needed. If reserve channel fail, the node should back off a random time $t^{\prime}$, then reserve channel again. Back off time t' should satisfy the following conditions:

1) t' should not be too long. Too long back off time may prolong access channel delay.

2) $t^{\prime}$ is random, and there is no relationship among the back off time of the neighboring nodes.

Here, we set $t^{\prime}$ is a random number in $[t, t+\Delta t]$.

Operation description of the method. In the single-hop broadcasting method based on busy tone, transmitter and receiver have two channels: the channel transmits data message is called data channel, and the other channel transmits busy tone is called busy tone channel. With an arbitrary node, the operation process of the broadcasting method is described in figure 1.

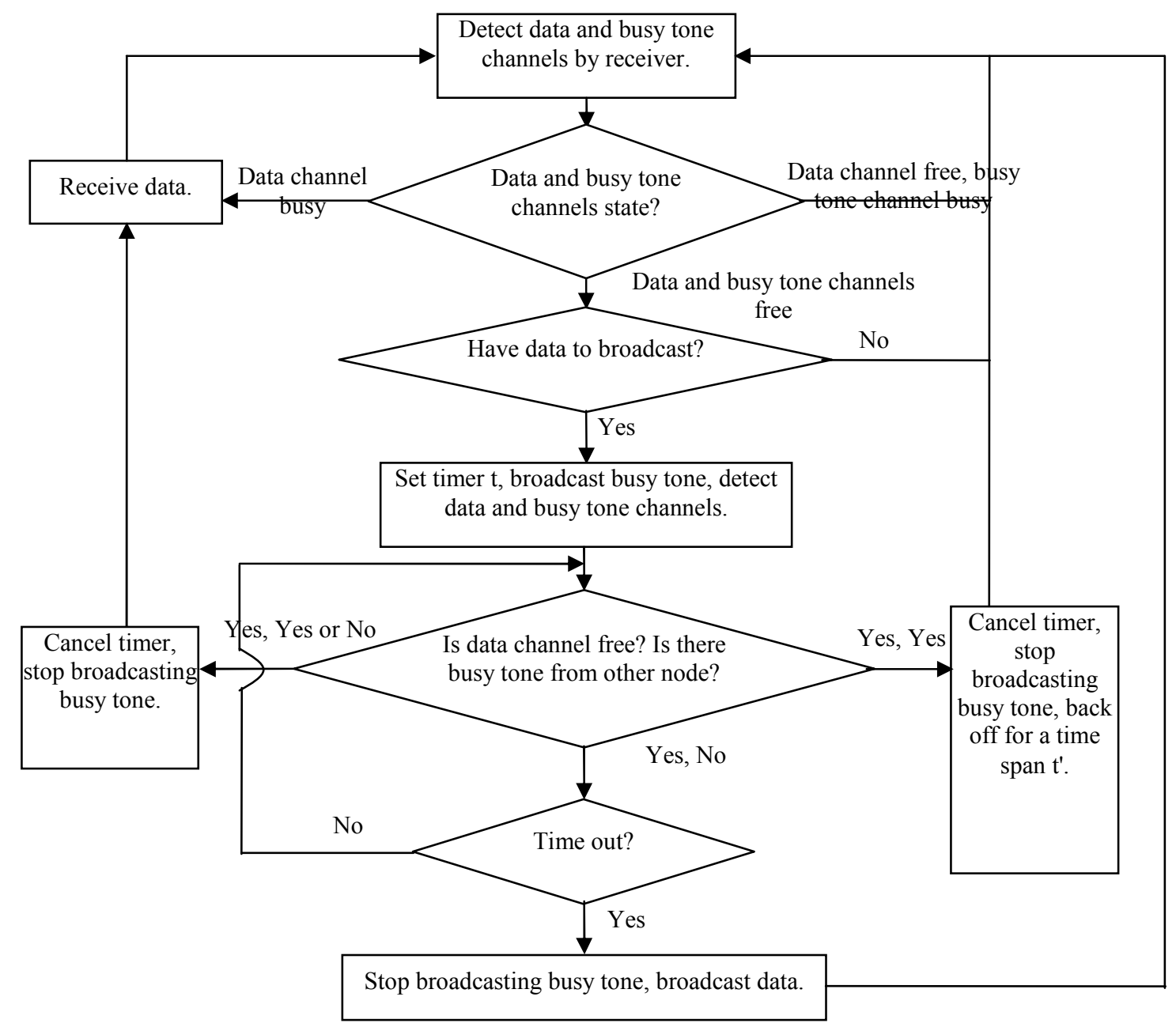

Figure 1. Operation process of the single-hop broadcasting method 
Following is the operation process of the single-hop broadcasting method:

(1) Node detects data channel and busy tone channel through receiver:

1) If data channel is busy, then receive data message. When receive data message is over, continue to detect data channel and busy channel.

2) If busy tone channel is busy and data channel is free, which means that there is other node in one hop range is reserving channel, continue to detect data and busy tone channel.

3) If data channel is free, busy tone channel is free, and the node itself has data to broadcast, then start to reserve channel.

4) If data channel is free, busy tone channel is free, and the node itself has no data to send, then continue to detect data and busy tone channel.

(2) Following is the process of channel reservation:

Set Timer $t$, broadcast busy tone, detect data and busy tone channels:

1) If data channel is busy before time out, then stop broadcast busy tone, and start to receive data message.

2) If data channel is free, and there's busy tone from other node before time out, then cancel timer, stop broadcasting busy tone, back off for a time span t', and continue to detect channel.

3) If data and busy tone channels are free before time out, the node reserves channel successfully. The node starts to broadcast data. When broadcast data message is over, continue to detect data and busy tone channel.

\section{Experiment Design and results}

To validate the performance of the single-hop broadcasting method, in this research, with OPNET network simulation platform, we modeled and implemented the broadcasting method. In the experiment, the following global statistics are collected.

(1) Average number of receiving nodes is the average amount of the nodes having received data packet correctly.

(2) Average end-to-end delay is the average time interval between the packet is generated and the packet is successfully received.

\section{Experiment of static network}

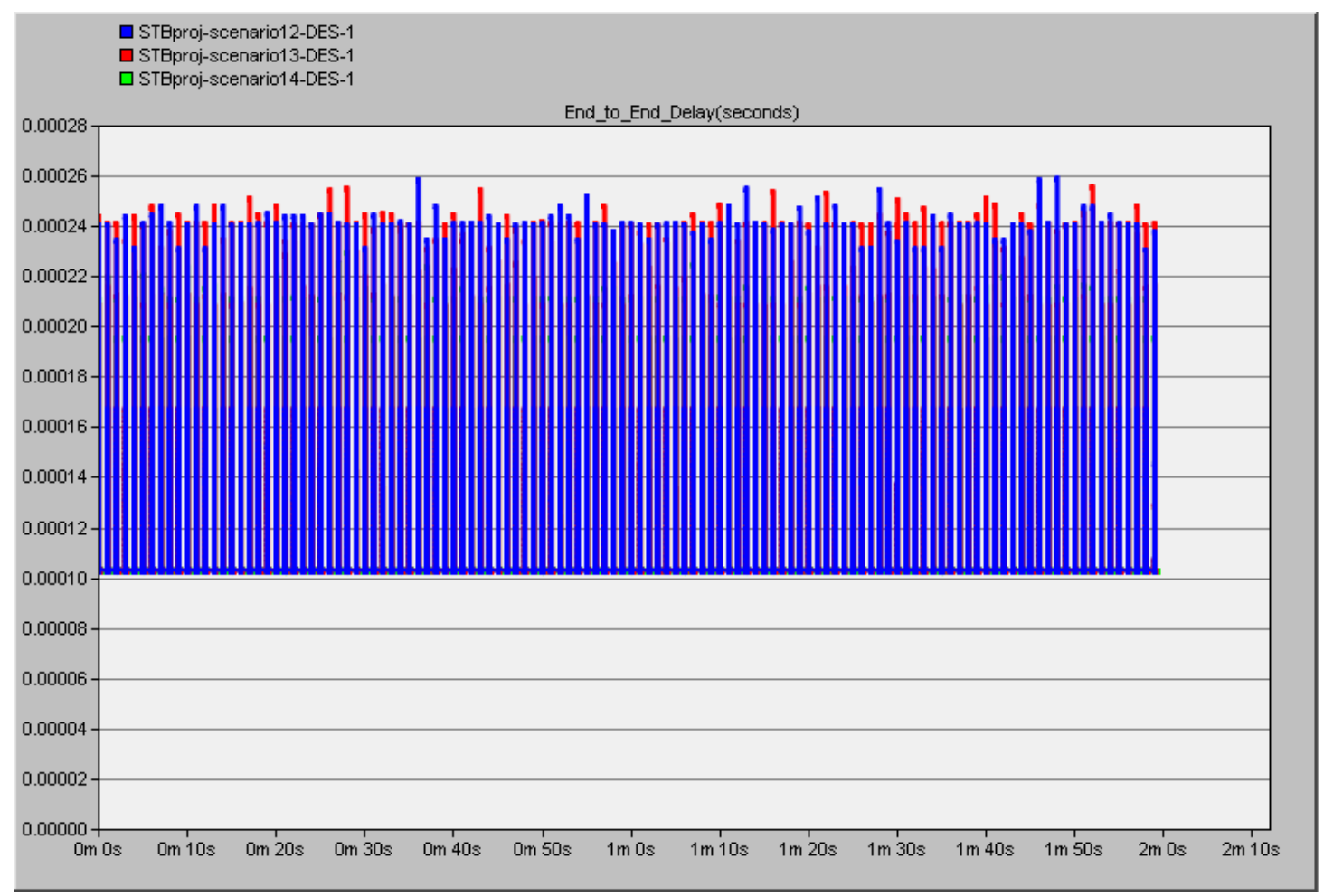

Figure 2. End-to-end delay of scenario 12 to scenario 14 
To validate the performance of the broadcasting method in the network with various load, a series of scenarios are designed. In all the scenarios, network range is $1200 \mathrm{~m} \times 50 \mathrm{~m}$, distance of one hop is $600 \mathrm{~m}$, data message length is $100 \mathrm{bit}$, data rate of transmitter and receivers is $10^{6} \mathrm{bps}$, interval time of node generating one data packet is 1 second. Nodes number of scenario 12, 13 and 14 are 40, 120, 240 respectively.

Experimental results are shown in figure 2 and table 1.

Table1. Average number of received nodes

\begin{tabular}{|ll|l|l|l|}
\hline No. of Scenario & 12 & 13 & 14 \\
\hline $\begin{array}{l}\text { Average Number } \\
\text { Received Node }\end{array}$ & of & 29 & 88 & 178 \\
\hline
\end{tabular}

As shown in figure 2 and table 1, in the network with various load, the end-to-end delay of the single-hop broadcasting method is stable and almost the same. The end-to-end delay is about $10^{-4}$ second, and most of the one hop range nodes could receive the broadcasted data.

Experiment of dynamic topology network. To validate the performance of the broadcasting method in the dynamic topology network, a series of scenarios are designed. In all the scenarios, network range is $3000 \mathrm{~m} \times 50 \mathrm{~m}$, nodes number is 65 , distance of one hop is $100 \mathrm{~m}$, data message length is 100bit, data rate of transmitter and receivers is $10^{6} \mathrm{bps}$, interval time of node generating one data packet is 1 second. Setup of scenario 19 to scenario 23 is list in table 2. Experimental results are shown in figure 3 and table 3.

Table 2. Setup of scenario 19 to scenario 23

\begin{tabular}{|l|l|l|}
\hline $\begin{array}{l}\text { No. } \\
\text { Scenario }\end{array}$ & Model of Movement & Moving velocity (m/s) \\
\hline 19 & All nodes move toward the same direction & 10 \\
\hline 20 & All nodes move toward the same direction & 50 \\
\hline 21 & Some nodes move, the other nodes is static & 10 \\
\hline 22 & All nodes move toward the same direction & {$[10,50]$} \\
\hline 23 & Nodes move in opposite direction & {$[10,50]$} \\
\hline
\end{tabular}

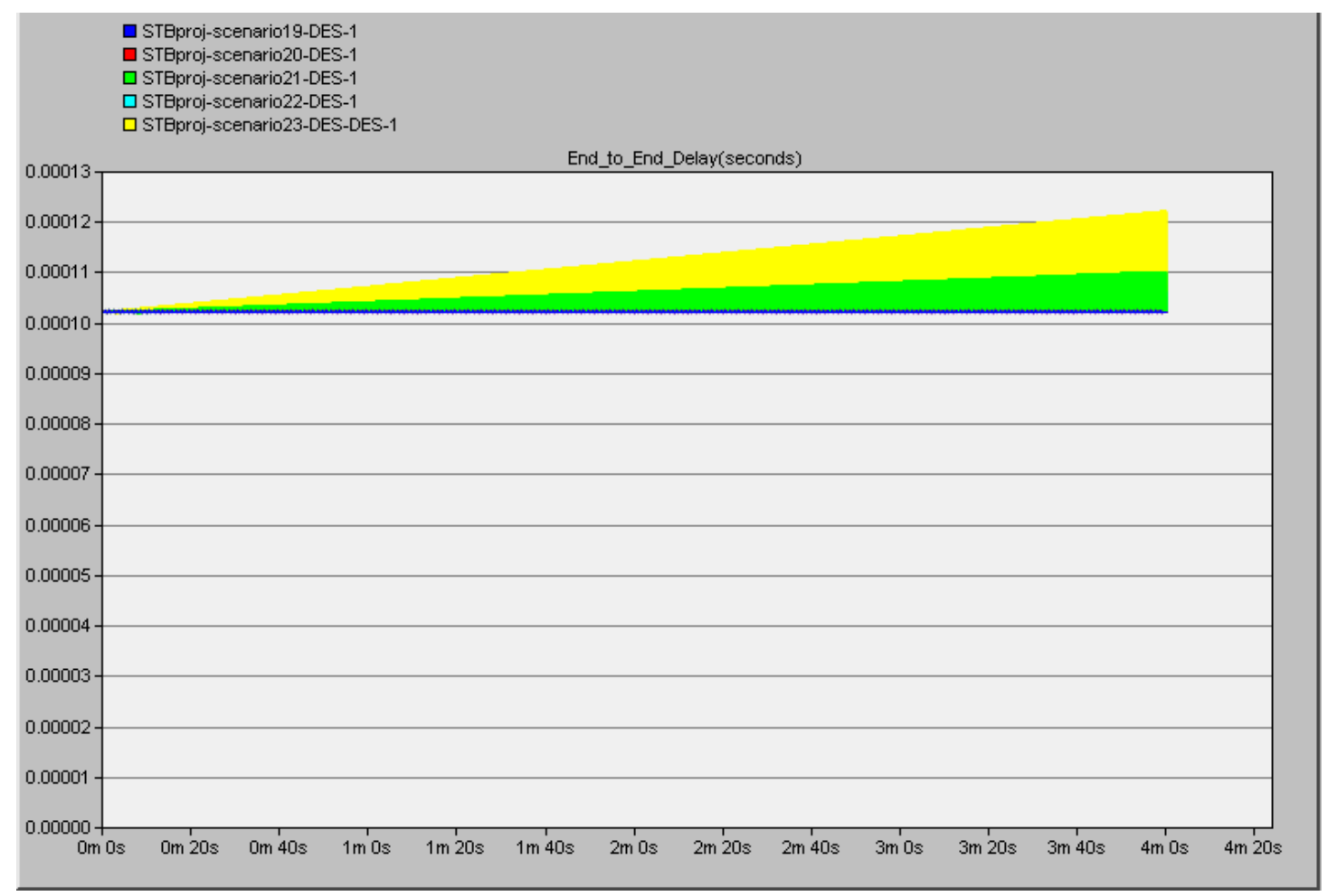

Figure 3. End-to-end delay of scenario 19 to scenario 23 
Table 3. Average number of received nodes

\begin{tabular}{|l|l|l|l|l|l|}
\hline No. of Scenario & 19 & 20 & 21 & 22 & 23 \\
\hline $\begin{array}{l}\text { Average Number of Received } \\
\text { Node }\end{array}$ & 7 & 7 & 7 & 7 & 7 \\
\hline
\end{tabular}

As shown in figure 3 and table 3, in dynamic topology network, the end-to-end delay is not stable. The more dynamics of the topology, the more end-to-end delay is. But the dynamic topology has no effect on the parameter of average number of received node.

\section{Summary}

In this paper, a single-hop broadcasting method based on busy tone is proposed. With OPNET network simulator, the method is modeled and implemented. Experimental results show that in the network with various load, performance of this broadcasting method is good. But this method is not applicable to the network with dynamic topology. This method should be improved further in the future research.

\section{References}

[1] Ken Tang, Mario Gerla, "Random Access MAC for Efficient Broadcast Support in Ad Hoc Networks," Proc. IEEE WCNC, 2000, pp. 454-459.

[2] Ken Tang, Mario Gerla. "MAC Reliable Broadcast in Ad Hoc Networks," Proc. IEEE MILCOM, 2001, pp. 1008-1013.

[3] Min-Te Sun, Lifei Huang, Anish Arora, Ten-Hwang Lai, "Reliable MAC Layer Multicast in IEEE 802.11 Wireless Networks,” Proc.ICPP, 2002, pp. 527-536.

[4] Toyserkani A.T..An Efficient Broadcast MAC Scheme for traffic safety applications in automotive networks. Wireless Communications and Networking Conference, 2006. WCNC 2006. IEEE. Volume:4. 3-6 April 2006.pp:2100-2105. 